\title{
Niveles séricos de marcadores inflamatorios en pacientes hipertensos de la E.S.E. Imsalud, Cúcuta, Colombia
}

Serum levels of inflammatory markers in hypertensive patients of E.S.E. Imsalud, Cucuta, Colombia

$\overline{\text { Y. Karina Ríos-Ramírez MSc', Sandra M. Mendoza-Toloza Bact }}{ }^{2}$, Johanna K. Carvajal-Ortega Bact², Viviana M. Taylor-Orozco PhD 3

Introducción: la hipertensión arterial es una condición clínica multifactorial, caracterizada por la elevación de la presión arterial sistólica o diastólica, establecida como un factor de riesgo importante en la aparición de enfermedades cardiovasculares. Objetivos: determinar los niveles séricos de IFN- $\gamma$, TNF- $\alpha$ y proteína C reactiva, relacionándolos con la hipertensión arterial en pacientes que asisten a la E.S.E. Imsalud-Unidad Materno Infantil la Libertad (Cúcuta, Colombia). Materiales y métodos: se realizó un estudio descriptivo, correlacional en individuos hipertensos y controles sanos seleccionados mediante encuesta estructurada. Los niveles séricos del IFN- $-\gamma$ y el TNF- $\alpha$ fueron analizados por ELISA y los de la proteína C reactiva ultrasensible por inmunoturbimetría. Resultados: el 52,8\% y el 29,3\% de los hipertensos presentaron concentraciones moderadamente altas de IFN- $y$ y TNF- $\alpha$ respectivamente. De acuerdo a los niveles séricos de proteína C reactiva ultrasensible, el 81,2\% de los hipertensos se ubicaron en riesgo cardiovascular bajo, el 6,2\% en medio y el $12,5 \%$ en alto. Conclusiones: los niveles elevados de IFN- $y$ en los pacientes hipertensos respecto a los controles sugieren que esta citoquina puede ser considerada un marcador útil como herramienta de evaluación del pronóstico de desarrollo de complicaciones cardiovasculares. Aunque el TNF- $\alpha$ no se encontró aumentado en los pacientes hipertensos, podría ser empleado en aquellos con dificil control de la enfermedad o en quienes no han iniciado el tratamiento antihipertensivo. La proteína C reactiva ultrasensible se reitera como un marcador útil en la estratificación de los niveles de riesgo en los hipertensos, abarcando desde bajo hasta alto de afectación cardiovascular.

Palabras clave: hipertensión, enfermedades cardiovasculares, factor de necrosis tumoral alfa, interferón gamma, proteína C-reactiva.

\footnotetext{
' Bacterióloga y Laboratorista Clínico, Msc. en Biología. Docente asistente e investigadora, Grupo de Investigación Biogen, programa de Bacteriología y Laboratorio clínico, Universidad de Santander. Cúcuta, Colombia. Correo electrónico: ye.rios@mail.udes.edu.co ${ }^{2}$ Bacterióloga y Laboratorista Clínico. Investigadora, Grupo de Investigación Biogen, programa de Bacteriología y Laboratorio clínico, Universidad de Santander. Cúcuta, Colombia.

${ }^{3}$ Bacterióloga y Laboratorista Clínico, MSc en Ciencias Básicas Biomédicas, PhD en Biología. Investigadora, Grupo de Investigación Microbiología Médica y Enfermedades Infecciosas, Universidad Santiago de Cali. Cali, Colombia.

Conflicto de intereses: las autoras declaran que no tienen conflicto de intereses Medicina \& Laboratorio 2015; 21: 483-492

Módulo 19 (Investigación), número 40. Editora Médica Colombiana S.A. $2015^{\odot}$

Recibido el 22 de septiembre de 2015; aceptado el 31 de octubre de 2015
}

Medicina \& Laboratorio Volumen 2I, Números 9-10, 2015. 
Introduction: Hypertension is a multifactorial clinical condition characterized by elevated systolic or diastolic blood pressure that it is establishing itself as a major risk factor in the onset of cardiovascular disease. Objectives: To determine serum levels of IFN- $\gamma$, TNF- $\alpha$ and C-reactive protein, relating to hypertension in patients attending the E.S.E. Imsalud - Unidad Materno Infantil La Libertad (Cucuta, Colombia). Material and methods: A correlational descriptive study was realized in hypertensive individuals and healthy controls selected through structured survey. Serum levels of IFN- $\gamma$ and TNF- $\alpha$ were analyzed by ELISA and high-sensitivity C-reactive protein levels by turbidimetric immunoassays. Results: The $52.8 \%$ and $29.3 \%$ of hypertensive subjects had moderately high concentrations of IFN- $\gamma$ and TNF- $\alpha$, respectively. According to serum levels of high-sensitivity C-reactive protein, $81.2 \%$ of hypertensive stood at low cardiovascular risk, $6.2 \%$ on average, and $12.5 \%$ at high risk. Conclusions: Elevated levels of IFN- $\gamma$ found in hypertensive patients respect to controls, suggest that this cytokine could be consider as a viable marker as a valuable tool in forecasting the development of cardiovascular complications. Although TNF- $\alpha$ not increased in hypertensive patients, could be used in those with difficult disease control or who have not initiated antihypertensive treatment. The high-sensitivity C-reactive protein can be reiterate as a marker useful in the stratification of the levels of risk in hypertensive patients, ranging from low to high risk of cardiovascular involvement.

Keywords: Hypertension, cardiovascular diseases, tumor necrosis factor-alpha, interferon-gamma, C-reactive protein.

Ríos-Ramírez YK, Mendoza-Toloza SM, Carvajal-Ortega JK, Taylor-Orozco VM. Niveles séricos de marcadores inflamatorios en pacientes hipertensos de la E.S.E. Imsalud, Cúcuta, Colombia. Medicina \& Laboratorio 2015; 21: 483-492.

Lcrót a hipertensión arterial es una condición clínica multifactorial caracterizada por la elevación crónica de la presión arterial igual o mayor que $140 \mathrm{mmHg}$ para la presión sistólica, igual o mayor que $90 \mathrm{mmHg}$ para la presión diastólica o ambas. Esta afección constituye una de las enfermedades crónicas más frecuentes que azotan a la humanidad, siendo generalmente silenciosa y lentamente progresiva, y presentándose en todas las edades, especialmente entre los 30 y 50 años $[1,2]$.

La elevación persistente de la presión arterial tiene una amplia repercusión en la salud pública, ya que se encuentra asociada a múltiples complicaciones degenerativas, frecuentemente a alteraciones funcionales o estructurales de órganos, generando problemas como el infarto agudo de miocardio, las enfermedades cerebrovasculares, la enfermedad renal crónica y las vasculopatías periféricas; además, es responsable directamente de un alto porcentaje de muertes [3]. El riesgo cardiovascular puede ser alto en personas con hipertensión leve asociado a otros factores de riesgo como la obesidad, el tabaquismo, la dieta inadecuada, el sedentarismo, la diabetes, la hipercolesterolemia, el nivel socioeconómico bajo y aquellos con antecedentes familiares previos [4].

Estudios experimentales y clínicos han mostrado que la activación inflamatoria está presente en el desarrollo de la hipertensión arterial y sus consecuencias cardiovasculares, participando en muchos procesos que contribuyen al desarrollo de la presión arterial elevada. Durante este proceso de activación se producen interacciones complejas entre diversos tipos de células que llevan a un aumento en la expresión de moléculas de adhesión, citoquinas, metaloproteasas de 
matriz y factores de crecimiento [5]. Una respuesta retardada y sostenida implica la presencia de factores inflamatorios como la proteína $C$ reactiva y de citoquinas como el factor de necrosis tumoral- $\alpha$ (TNF- $\alpha$ ) y el interferón- $\nu$ (INF- $\gamma)[6,7]$, que pueden servir para ayudar a identificar a los pacientes en riesgo de enfermedad cardiovascular, así como para vigilar la eficacia de los tratamientos $[6,8]$.

Cada día se hace más evidente que las alteraciones metabólicas que condicionan a un conjunto de enfermedades sistémicas, dentro de las cuales se encuentran las cardiovasculares, necesitan una mayor comprensión para su alcance clínico y un enfoque terapéutico más adecuado. En el caso de la hipertensión arterial el control debe ser oportuno para evitar las apariciones de ciertas complicaciones cardiacas, del sistema nervioso central, renales y retinianas, que pueden perjudicar seriamente la salud de quienes la padecen. Por lo tanto, el objetivo de esta investigación fue determinar el grado de relación entre los niveles séricos del INF- $\gamma$, el TNF- $\alpha$ y la proteína C reactiva en pacientes diagnosticados con hipertensión arterial y que fueron atendidos en la E.S.E. Imsalud-Unidad Materno Infantil la Libertad (Cúcuta, Colombia) para demostrar su utilidad como marcadores pronósticos de daño cardiovascular temprano en esta población, de modo que sirvan como herramienta para alertar al médico en la toma de decisiones o conductas a seguir necesarias para el control e instauración del tratamiento adecuado en estos individuos.

\section{Materiales y métodos}

\section{Tipo y población de estudio}

Se realizó un estudio descriptivo-correlacional con base en los datos clínicos y análisis del laboratorio. Los datos fueron recolectados mediante encuesta estructurada con el propósito de recopilar información personal relevante, como las variables sociodemográficas (edad y sexo), el consumo de medicamentos, el hábito de fumar, la ingesta de alcohol, la realización de ejercicio físico y el padecimiento de alguna enfermedad adicional a la hipertensión, y antecedentes familiares de hipertensión arterial, diabetes mellitus, obesidad, dislipidemia, híper o hipotiroidismo, nefropatía, infarto agudo al miocardio y enfermedad cerebrovascular. También se preguntó por información sobre la dieta y los conocimientos de los posibles riesgos de padecer hipertensión arterial.

Como criterios de inclusión se establecieron individuos mayores de 18 años, de ambos sexos, que aceptaron de manera voluntaria participar en el estudio y de los cuales se obtuvo un consentimiento informado por escrito y una aprobación para el almacenamiento y uso futuro de sus muestras. Como criterios de exclusión la presencia de enfermedades diferentes a la hipertensión arterial y que aumentaran el riesgo cardiovascular, entre las que se consideraron la diabetes mellitus, la dislipidemia, la insuficiencia renal crónica, la enfermedad obstructiva crónica, la tuberculosis, el asma, el cáncer, la retinopatía, el infarto agudo de miocardio, la insuficiencia cardiaca, la enfermedad cardiovascular y la enfermedad arterial periférica. Adicional a esto, se excluyeron los individuos con presencia de algún proceso de tipo infeccioso como por ejemplo chikunguña o gripe, dadas las características de la población en general en el momento del estudio.

A todos los individuos incluidos en la investigación se les midió la presión arterial, la frecuencia cardiaca, el peso y la talla, y se recolectaron muestras sanguíneas en ayunas por punción venosa para la obtención de suero mediante centrifugación. Los sueros de cada uno de los participantes fueron 
separados y alicuotados en dos viales debidamente marcados; una de las alícuotas se almacenó a -20 ${ }^{\circ} \mathrm{C}$ para los análisis posteriores de citoquinas y proteína $\mathrm{C}$ reactiva ultrasensible y la otra se destinó para la medición de parámetros adicionales como la glicemia y el perfil lipídico, los cuales debían encontrarse dentro del rango normal de valores para descartar otras condiciones concomitantes consideradas en los criterios de exclusión, que pudiesen interferir con los resultados.

Para el grupo de estudio se eligieron 17 pacientes con diagnóstico previo de hipertensión arterial de bajo riesgo cardiovascular y que asistían al club de hipertensos de la E.S.E. Imsalud-Unidad Materno Infantil la Libertad, lugar donde se llevó a cabo la toma de muestra, y I 5 individuos sanos que no asistían a esta institución, cuyas muestras fueron tomadas en sus respectivos domicilios.

Todos los consentimientos y procedimientos fueron avalados por el comité de ética de la E.S.E. Imsalud.

\section{ELISA para TNF- $\alpha$ e IFN- $\gamma$}

Las mediciones de los niveles de TNF- $\alpha$ e IFN- $\gamma$ a partir de los sueros de los pacientes alicuotados previamente y almacenados a $-20{ }^{\circ} \mathrm{C}$, se analizaron utilizando los estuches de análisis inmunoenzimático de alta sensibilidad Human TNF alpha ELISA Ready-SET-Go! y Human IFN gamma ELISA Ready-SET-Go! (eBioscience, Inc., San Diego, California, Estados Unidos). Cada muestra se valoró por duplicado usando un único plato por ensayo y las densidades ópticas se determinaron usando el lector de microplatos ChroMate ${ }^{\circledR} 4300$ (Awareness Technology, Inc., Palm City, Florida, Estados Unidos) a una longitud de onda de $450 \mathrm{~nm}$ y filtro diferencial de $630 \mathrm{~nm}$, para ambas citoquinas.

\section{Ensayo inmunoturbidimétrico para proteína C reactiva ultrasensible}

La determinación cuantitativa de la proteína $C$ reactiva ultrasensible se realizó mediante el método inmunoturbidimétrico con látex de la Línea Turbitest AA PCR ultrasensible (Wiener Laboratorios S.A.I.C., Rosario, Argentina) por medio del analizador para proteína $C$ reactiva ultrasensible CB 350i de la misma casa comercial, a una longitud de onda de 570 nm.

\section{Análisis estadístico}

Las técnicas utilizadas en el análisis y procesamiento de los datos fueron cuantitativas; para ello se codificaron los datos obtenidos y se tabularon en tablas, y a partir de estos se realizó un análisis multivariado aplicando la prueba de ANOVA de una vía con análisis de contraste de hipótesis mediante prueba de Dunnett, el coeficiente de correlación de Spearman, la prueba t de Student y la prueba $U$ de Mann-Whitney, utilizando el paquete estadístico GraphPad Prism 6 (GraphPad Software, Inc., La Jolla, California, Estados unidos). Los valores de $p<0,0$ I se aceptaron como estadísticamente significativos.

\section{Resultados}

\section{Características generales de la población estudiada}

Los datos recopilados en la encuesta de los 17 pacientes hipertensos (grupo de estudio) y los 15 individuos sanos (grupo control), con su respectiva media y significancia estadística se resumen en la tabla I. La proporción hallada de hombres y mujeres del grupo de hipertensos fue ocho 
(47, I\%) y nueve (52\%), respectivamente, y en el grupo control fue siete $(46,6 \%)$ y ocho $(53,4 \%)$, respectivamente. De igual manera, el promedio de peso, índice de masa corporal (IMC) y frecuencia cardiaca obtenida fue muy similar entre ambos grupos. La edad promedio obtenida de los dos grupos fue de 60 años, con un rango entre 39 y 88 años. La cifra promedio de la presión arterial fue de 127/78, encontrándose más elevada en los individuos hipertensos (1 34/83) a pesar del régimen terapéutico, en comparación con el grupo control ( I 19/73) (véase tabla I).

\begin{tabular}{|c|c|c|c|c|}
\hline Parámetro & Grupo control $(n=15)$ & Grupo hipertensos $(n=17)$ & Valor $p$ & Total $(n=32)$ \\
\hline \multicolumn{5}{|c|}{ Características personales [media \pm desviación estándar (rango)] } \\
\hline Edad (años) & $56 \pm 11,6(43-88)$ & $64 \pm 12,7(39-88)$ & 0,0835 & $60,4 \pm 12,8(39-88)$ \\
\hline $\begin{array}{l}\text { Sexo }[\mathrm{n}(\%)] \\
\text { Masculino } \\
\text { Femenino }\end{array}$ & $\begin{array}{l}7(46,6) \\
8(53,4)\end{array}$ & $\begin{array}{l}8(47,1) \\
9(52,9)\end{array}$ & 0,6667 & $\begin{array}{l}15(46,8) \\
17(53,2)\end{array}$ \\
\hline Peso $(\mathrm{Kg})$ & $67,3 \pm 10,4(49,1-92,0)$ & $68,6 \pm 10,5(49,5-84,0)$ & 0,6614 & $68,0 \pm 10,5(49,1-92,0)$ \\
\hline IMC $\left(\mathrm{Kg} / \mathrm{cm}^{2}\right)$ & $26,0 \pm 3,7(20,8-35,9)$ & $26,8 \pm 4,3(19,3-35,4)$ & 0,6213 & $26,5 \pm 4(19,3-35,9)$ \\
\hline $\begin{array}{l}\text { Frecuencia } \\
\text { cardiaca (latido/ } \\
\text { minuto) }\end{array}$ & $72,4 \pm 10,6(56-96)$ & $73,9 \pm 9,2(60-96)$ & 0,6464 & $73,2 \pm 9,9(56-96)$ \\
\hline $\begin{array}{l}\text { Presión arterial } \\
(\mathrm{mmHg}) \\
\text { Sistólica } \\
\text { Diastólica }\end{array}$ & $\begin{array}{l}119 \pm 3,8 \\
73 \pm 8,5\end{array}$ & $\begin{array}{l}134 \pm 20,7 \\
83 \pm 12,6\end{array}$ & $\begin{array}{l}0,0026 * * \\
0,0153 *\end{array}$ & $\begin{array}{l}127 \pm 16,9 \\
78 \pm 12\end{array}$ \\
\hline \multicolumn{5}{|c|}{ Antecedentes familiares [n (\%)] } \\
\hline $\begin{array}{l}\text { Hipertensión } \\
\text { arterial }\end{array}$ & $4(26,6)$ & $10(58,8)$ & 0,0870 & $14(43,7)$ \\
\hline $\begin{array}{l}\text { Diabetes } \\
\text { mellitus }\end{array}$ & $4(26,6)$ & $5(29,4)$ & 0,0886 & $9(28,1)$ \\
\hline $\begin{array}{l}\text { Enfermedad } \\
\text { coronaria }\end{array}$ & $2(13,3)$ & $6(35,2)$ & $0,4 \mid 88$ & $8(25)$ \\
\hline $\begin{array}{l}\text { Infarto agudo de } \\
\text { miocardio }\end{array}$ & I $(6,6)$ & $4(23,5)$ & 0,3382 & $5(15,6)$ \\
\hline Obesidad & $3(20)$ & I $(5,8)$ & 0,3192 & $3(12,5)$ \\
\hline $\begin{array}{l}\text { Enfermedad } \\
\text { vascular }\end{array}$ & $3(20)$ & I $(5,8)$ & 0,3192 & $3(12,5)$ \\
\hline Dislipidemia & I $(6,6)$ & I $(5,8)$ & 1,0 & $2(6,2)$ \\
\hline Hipertiroidismo & $0(0)$ & $2(11,7)$ & 0,4859 & $2(6,2)$ \\
\hline $\begin{array}{l}\text { * Valor } p \leq 0,05 \\
\text { ** Valor } p \leq 0,01\end{array}$ & & & & \\
\hline
\end{tabular}

Del total de la muestra estudiada $(n=32)$ se observó que el 43,7\% presentaba antecedentes familiares de hipertensión arterial, seguido de un 28, $1 \%$ para diabetes mellitus, 25\% para enfermedad coronaria, 15,6\% para infarto aguda de miocardio y 12,5\% tanto para obesidad como para enfermedad vascular. El 58,8\% de los hipertensos que conformaron el grupo de estudio mostraron antecedentes familiares para esta entidad mientras que en el grupo control el porcentaje fue de $26,6 \%$ (véase tabla I). En cuanto a los medicamentos más utilizados para el manejo de la hipertensión en el grupo de estudio se destacaron en su orden: losartán (70,5\%), aspirina (52,9\%), amlodipino (29,4\%), hidroclorotiazida (29,4\%) y enalapril $(23,5 \%)$. Además, se encontró que en el 
grupo de hipertensos las combinaciones más comunes entre los medicamentos fueron: losartán/ aspirina en un $35,2 \%$, losartán/amlodipino en un $29,4 \%$, y losartán/hidroclorotiazida en un 23,5\%.

\section{Niveles de TNF- $\alpha$, IFN- $\gamma$ y proteína C reactiva ultrasensible}

En la tabla 2 se muestra el valor medio y el rango intercuartílico de las concentraciones séricas de IFN- $\gamma$, TNF- $\alpha$ y proteína $C$ reactiva en el suero de los individuos del grupo de estudio (hipertensos) y el grupo control. Los resultados mostraron una diferencia estadísticamente significativa entre el grupo de hipertensos y el grupo control en los niveles de IFN- $\gamma(p<0,0$ I). Por otra parte, el TNF- $\alpha$ y la proteína $C$ reactiva no fueron significativamente diferentes entre los dos grupos $(p>0,0 \mathrm{I})$.

\begin{tabular}{|c|c|c|c|c|}
\hline Parámetro & Grupo control $(n=15)$ & Grupo hipertensos $(n=17)$ & Valor $\mathrm{p}$ & Total $(n=32)$ \\
\hline $\mathrm{IFN}-\boldsymbol{\gamma}(\mathrm{pg} / \mathrm{mL})$ & $17,8 \pm 50,9$ & $65,5 \pm 32,7$ & 0,000 I ******* & $44,0 \pm 48,2$ \\
\hline TNF- $\alpha(p g / m L)$ & $5,8 \pm 2,7$ & $4,7 \pm 2,3$ & 0,3052 & $5,2 \pm 2,6$ \\
\hline $\begin{array}{l}\text { Proteína C reactiva } \\
\text { ultrasensible }(\mathrm{mg} / \mathrm{L})\end{array}$ & $1,7 \pm 2,3$ & $1,2 \pm 2,7$ & 0,4212 & $1,4 \pm 2,5$ \\
\hline ******* Valor $p \leq 0,000$ & & & & \\
\hline
\end{tabular}

En la tabla 3 se muestra el coeficiente de correlación $\mathrm{R}$ y el valor $p$ de los niveles séricos de IFN- $\gamma$, TNF- $\alpha$ y proteína $C$ reactiva de los pacientes hipertensos y los controles analizados estadísticamente mediante Spearman. El análisis demostró en los hipertensos una correlación significativa entre los valores de la proteína $C$ reactiva ultrasensible y el IFN- $\gamma$, pero no entre los valores de TNF- $\alpha$ e IFN- $\gamma$ y del TNF- $\alpha$ y la proteína $C$ reactiva ultrasensible. Entretanto, no se encontró correlación significativa entre los biomarcadores determinados en el grupo control.

\begin{tabular}{|c|c|c|c|c|c|c|c|}
\hline \multirow[t]{2}{*}{ Relación } & \multirow[t]{2}{*}{ Valor } & \multicolumn{3}{|c|}{ Hipertensos } & \multicolumn{3}{|c|}{ Controles } \\
\hline & & IFN- $\gamma$ & TNF- $\alpha$ & $\begin{array}{l}\text { Proteína } C \text { reactiva } \\
\text { ultrasensible }\end{array}$ & IFN- $\gamma$ & TNF- $\alpha$ & $\begin{array}{l}\text { Proteína } C \text { reactiva } \\
\text { ultrasensible }\end{array}$ \\
\hline IFN- $\gamma$ & $\begin{array}{l}\mathrm{R} \\
\mathrm{p}\end{array}$ & -- & $\begin{array}{l}0,256 \\
0,317\end{array}$ & $\begin{array}{l}-0,47 \mid \\
0,057\end{array}$ & -- & $\begin{array}{l}-0,018 \\
0,952\end{array}$ & $\begin{array}{l}0,047 \\
0,871\end{array}$ \\
\hline TNF- $\alpha$ & $\begin{array}{l}\mathrm{R} \\
p\end{array}$ & $\begin{array}{l}0,256 \\
0,317\end{array}$ & -- & $\begin{array}{l}-0,282 \\
0,271\end{array}$ & $\begin{array}{l}-0,018 \\
0,952\end{array}$ & -- & $\begin{array}{l}0,033 \\
0,906\end{array}$ \\
\hline $\begin{array}{l}\text { Proteína C reactiva } \\
\text { ultrasensible }\end{array}$ & $\begin{array}{l}\mathrm{R} \\
\mathrm{p}\end{array}$ & $\begin{array}{l}-0,471 \\
0,057\end{array}$ & $\begin{array}{l}-0,282 \\
0,271\end{array}$ & -- & $\begin{array}{l}0,047 \\
0,871\end{array}$ & $\begin{array}{l}0,033 \\
0,906\end{array}$ & -- \\
\hline
\end{tabular}

En la figura I se muestran los niveles séricos de los marcadores analizados. En el 52,8\% de los hipertensos se presentaron concentraciones entre moderadas y elevadas de IFN- $\gamma$, mientras que el TNF- $\alpha$ se encontró en el 70,5\% en concentraciones ligeramente elevadas, en el 17,6\% moderadas y en el I I,7\% elevadas. En cuanto a la proteína $C$ reactiva se clasificó de acuerdo al riesgo de desarrollo de un evento cardiovascular en riesgo bajo, medio y alto. De esta manera, en el grupo de hipertensos el $81,2 \%$ de ellos se encontró en riesgo bajo, el 6,2\% en riesgo medio y el $12,5 \%$ en riesgo alto (véase figura I). 
A

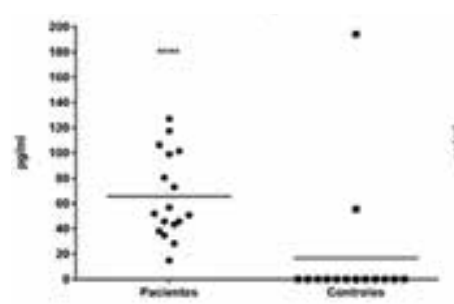

B

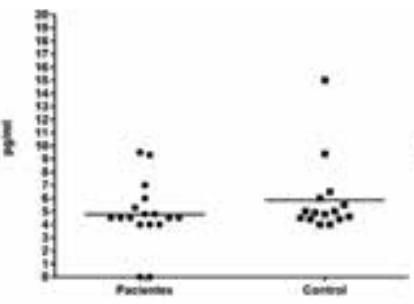

(C)

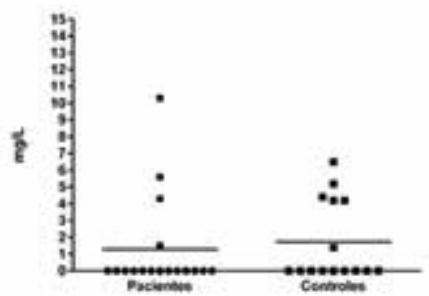

Figura I. Niveles de IFN- $\gamma(A)$, TNF- $\alpha$ (B) y proteína $C$ reactiva ultrasensible (C) determinados mediante ensayo por duplicado, al que se le aplicó la prueba t de Student y la prueba $U$ de Mann-Whitney para determinar las diferencias significativas entre los pacientes (hipertensos) y los controles (sanos).

\section{Discusión}

La hipertensión arterial es una afección crónica muy frecuente en la población adulta a nivel mundial, que constituye el principal factor de riesgo para el desarrollo de enfermedad cerebrovascular, coronaria, arterioesclerótica y renal; encontrándose entre las más importantes causas de muerte en los países desarrollados y en la mayor parte de los países en vías de desarrollo [9]. Algunos de los aspectos abarcados de los individuos incluidos en este estudio fueron la edad, el género, la presión arterial y los antecedentes familiares, encontrando en el grupo de hipertensos un promedio de edad de 64 años, mayor afectación por esta condición en las mujeres y sin mayores diferencias en cuanto a la edad y el género respecto al grupo control; de manera similar a lo encontrado por Alvarado y colaboradores (2012) [I0] y Tissert y colaboradores (20|4) [I I].

En cuanto a la presión arterial sistólica y diastólica la media obtenida fue de 134/83. Específicamente, el 23,5\% de los hipertensos mostraron presión arterial sistólica por encima de 140 mmHg, el 17,6\% presión arterial diastólica por encima de 90 mmHg y el 17,6\% ambas medidas alteradas. Respecto a los individuos sanos se hallaron diferencias estadísticamente significativas en cuanto a la presión arterial sistólica y la presión arterial diastólica. Estos resultados pueden ser comparables a los obtenidos por Manimunda y colaboradores (20l I) [12], quienes, al analizar un grupo de individuos hipertensos, encontraron un mayor porcentaje de afectación tanto de la presión arterial sistólica como de la diastólica.

Respecto a los antecedentes familiares de hipertensión arterial el 58,8\% de los pacientes hipertensos tenían un familiar afectado; cifra que es muy similar a la encontrada en el estudio de Doğan y colaboradores (2012) [13]. Los antecedentes familiares no solo son útiles para evaluar la predisposición a padecer la hipertensión arterial, sino que ayudan a comprender las diversas características de su manifestación clínica como la edad de aparición, la cual determina la gravedad con la que dicha enfermedad se desarrolla en el paciente. Esto lleva a pensar que no solo se debe prestar atención a la salud del paciente hipertenso, sino que se debe vigilar su núcleo familiar para lograr intervenir oportunamente y minimizar al máximo las complicaciones que pueden surgir al padecer esta enfermedad [14]. 
En la etiología de la enfermedad cardiovascular han sido implicadas múltiples vías biológicas dentro de las que se incluye la inflamación y el estrés oxidativo [15]. En varios estudios se ha reconocido que la inflamación de bajo grado desempeña un papel fisiopatológico crucial en la hipertensión y en la enfermedad cardiovascular; participando en procesos que contribuyen al desarrollo de la presión arterial elevada $[16,17]$. Durante la hipertensión se produce la liberación de ciertas moléculas como el TNF- $\alpha$, interleuquinas, linfoquinas, monoquinas, interferones, factores estimulantes de colonias y factores de crecimiento transformante, provocando, en determinadas situaciones, daño en las células sanas del individuo [18].

El TNF- $\alpha$ desempeña un papel principal en el desarrollo de trastornos cardiovasculares; de hecho, el aumento de sus concentraciones plasmáticas se ha encontrado en pacientes con enfermedad coronaria prematura, infarto agudo de miocardio, enfermedad arterial periférica e insuficiencia cardíaca congestiva [19]. Esta molécula tiene la capacidad de deprimir la contractilidad cardíaca, aumentar el catabolismo proteico y producir disfunción endotelial, inflamación, dilatación, fibrosis e hipertrofia cardíaca, activación neurohormonal y apoptosis de los cardiomiocitos [20]. En el presente estudio se encontró que todos los pacientes hipertensos tenían concentraciones elevadas del TNF- $\alpha$, la mayoría de ellos ligeramente incrementadas; sin embargo, no se observaron diferencias estadísticamente significativas en los niveles de este marcador entre los individuos del mismo grupo ni en los hipertensos respecto a los controles. Este hallazgo lleva a pensar que los niveles de TNF- $\alpha$ en estos pacientes se encuentran influenciados por el tratamiento antihipertensivo, tal como fue reportado por Li y colaboradores (2006) [2I]

En este trabajo se hallaron diferencias estadísticas significativas en los valores de IFN- $\gamma$ entre el grupo de pacientes hipertensos y el grupo control, con una correlación significativa entre los valores de esta citoquina y los de la proteína $C$ reactiva ultrasensible, pero no con los de TNF- $\alpha$. Partiendo de los resultados obtenidos los niveles de IFN- $\gamma$ podrían ser los mejores predictores de un evento cardiovascular futuro en la población hipertensa, ya que muy posiblemente los fármacos utilizados para el tratamiento de esta entidad no estarían ejerciendo acción alguna sobre los niveles de esta citoquina, revelando las condiciones reales en la que estaría evolucionando un individuo con hipertensión arterial. Actualmente son pocos los estudios realizados en seres humanos que asocien o involucren el IFN- $\gamma$ en el desarrollo de eventos cardiovasculares en la población hipertensa. La mayoría de los avances obtenidos han sido realizados en ratas, en las que se ha encontrado una asociación fuertemente positiva entre la afectación cardíaca y el aumento en las concentraciones de esta citoquina, tal como se menciona en el trabajo de revisión de Levick y Goldspink (2014) [22].

La proteína $C$ reactiva, entre todos los marcadores inflamatorios circulantes del proceso aterosclerótico, ha recibido la mayor atención, sobre todo debido a su fácil medición como analito [23]. Por sí sola, la proteína $C$ reactiva tiene la capacidad de predecir futuros eventos cardiovasculares en sujetos sanos y en pacientes con enfermedad cardiovascular establecida, constituyéndose no solo un marcador de enfermedad cardíaca, sino también un agente proaterogénico y proinflamatorio $[9,24]$. Hoy día se pueden detectar los niveles de proteína $C$ reactiva ultrasensible requeridos para la predicción del riesgo cardiovascular. La Asociación Americana de Cardiología (AHA; del inglés, American Heart Association), utilizando este método, recomienda interpretar los niveles por debajo de I,0 mg/L como 
riesgo bajo, entre I, I mg/L ay 3,0 mg/L como riesgo moderado y entre 3,I mg/L a I0,0 mg/L como riesgo alto [25]. En esta investigación los niveles séricos de proteína $C$ reactiva en los individuos hipertensos permiten clasificarlos en un riesgo cardiovascular bajo; sin embargo, no se encontraron diferencias estadísticamente significativas en los niveles de este marcador entre los individuos hipertensos y los del grupo control; hallazgos que concuerdan con los previamente reportados por Bautista y colaboradores en 2005 [26].

\section{Conclusiones}

De acuerdo a lo encontrado en esta investigación el TNF- $\alpha$, el IFN- $\gamma$ y la proteína $C$ reactiva ultrasensible podrían ser biomarcadores de gran importancia en el control, evaluación y pronóstico de la población hipertensa; siendo el IFN- $\gamma$ el más destacado. El TNF- $\alpha$ podría ser útil en el control farmacológico, encontrándose aumentado en individuos resistentes al tratamiento, y la proteína C reactiva ultrasensible, por su parte, reafirma su capacidad como evaluador del riesgo a padecer un evento cardiovascular futuro.

La principal limitación del estudio se vio reflejada en el tamaño de la muestra utilizada, como consecuencia a la dificultad para encontrar individuos que cumplieran con los criterios de inclusión planteados. Esto se debió en buena medida a las características epidemiológicas de la población al momento de la ejecución de la investigación. Por tal motivo, a pesar de que los resultados aquí presentados son promisorios, se hace necesario reproducir el estudio con una mayor cantidad de participantes que otorguen la significancia estadística necesaria para ratificar el potencial del TNF- $\alpha$, el IFN- $\gamma$ y la proteína $C$ reactiva ultrasensible como marcadores pronósticos de enfermedad cardiovascular en individuos hipertensos.

\section{Agradecimientos}

Las investigadoras desean agradecer a la E.S.E. Imsalud y a las Bacteriólogas Nancy Bustos y Jhoalmis Sierra por su colaboración.

\section{Bibliografía}

I. Pinilla AE, Barrera MP, Agudelo JF, Agudelo C, Pardo R, Gaitán H, et al. Guía 13: Guía de atención de la hipertensión arterial. In: República de Colombia, Ministerio de la Protección Social, Programa de Apoyo a la Reforma de Salud, Universidad Nacional de Colombia, Instituto de Investigaciones Públicas eds. Guías de promoción de la salud y prevención de enfermedades en la salud pública. Bogotá, Colombia: Programa de Apoyo a la Reforma de Salud - PARS, Ministerio de la Protección Social - MPS; 2007: 151-218.

2. Mankin LA. Update in Hypertension Therapy. Med Clin North Am 20 16; 100: 665-693.

3. Gamboa R, Rospigliosi A. Más allá de la hipertensión arterial. Acta Méd Peruana 20 10; 27: 45-52.
4. Organización Mundial de la Salud. Información general sobre la hipertensión en el mundo: una enfermedad que mata en silencio, una crisis de salud pública mundial. Ginebra, Suiza; 2013.

5. McKay HS, Bream JH, Margolick JB, Martinez-Maza O, Phair JP, Rinaldo CR, et al. Host factors associated with serologic inflammatory markers assessed using multiplex assays. Cytokine 20।6; 85: 7I-79.

6. Schiffrin EL. The immune system: role in hypertension. Can J Cardiol 20 13; 29: 543-548.

7. Signorelli SS, Fiore V, Malaponte G. Inflammation and peripheral arterial disease: the value of circulating biomarkers (Review). Int J Mol Med 20।4; 33: 777-783. 
8. Mirhafez SR, Zarifian A, Ebrahimi M, Ali RF, Avan A, Tajfard M, et al. Relationship between serum cytokine and growth factor concentrations and coronary artery disease. Clin Biochem 20I5; 48: 575-580.

9. Pérez MD, León JL, Fernández MA. El control de la hipertensión arterial: un problema no resuelto. Rev Cubana Med 201 1; 50: 31 I-323.

10. Alvarado C, Jaramillo M, Matijasevic E, Rendón ID, Quitian J. Estudio poblacional de factores de riesgo cardiovascular relacionados con el estilo de vida, hallazgos electrocardiográficos y medicación actual de pacientes valorados por el servicio de Cardiología. Rev Colomb Cardiol 2012; 19: 61-71.

II. Tissert M, Soltura LU, Tissert HA, Tissert HI. Características de los pacientes diagnosticados con hipertensión arterial de la comunidad del policlínico "Josué País García». I6 de abril 20।4; 53: 55-63.

12. Manimunda SP, Sugunan AP, Benegal V, Balakrishna N, Rao MV, Pesala KS. Association of hypertension with risk factors \& hypertension related behaviour among the aboriginal Nicobarese tribe living in Car Nicobar Island, India. Indian J Med Res 20 I I; I 33: 287-293.

13. Doğan N, Toprak D, Demir S. Hypertension prevalence and risk factors among adult population in Afyonkarahisar region: a cross-sectional research. Anadolu Kardiyol Derg 2012; 12: 47-52.

14. Álvarez J, Lurbe E. Influencia de los antecedentes familiares sobre la edad de aparición de la hipertensión. Implicación de la impronta genética. Hipertens Riesgo Vasc 2008; 25: 225-227.

15. Stoner L, Lucero AA, Palmer BR, Jones LM, Young JM, Faulkner J. Inflammatory biomarkers for predicting cardiovascular disease. Clin Biochem 2013; 46: |353-137|.

16. Alonso-Rodríguez D, Moreno-Téllez E, Alarcón-Martínez Y, Pedroso-Filiberto E. Proteína $C$ reactiva como marcador de inflamación en hipertensión arterial aguda. Rev Med Inst Mex Seguro Soc 201 I; 49: 345-347.
17. Virdis A, Dell'Agnello U, Taddei S. Impact of inflammation on vascular disease in hypertension. Maturitas 2014; 78: 179-183.

18. Sprague AH, Khalil RA. Inflammatory cytokines in vascular dysfunction and vascular disease. Biochem Pharmacol 2009; 78: 539-552.

19. Xiao H, Liao YH, Chen ZJ. Tumor necrosis factor-alpha: a new mechanism of ischemic ventricular fibrillation? Chin Med J (Engl) 2008; 121: |848-|85|.

20. Gullestad L, Ueland T, Vinge LE, Finsen A, Yndestad A, Aukrust P. Inflammatory cytokines in heart failure: mediators and markers. Cardiology 2012; 122: 23-35.

21. Li B, Liao YH, Cheng X, Ge H, Guo H, Wang M. Effects of carvedilol on cardiac cytokines expression and remodeling in rat with acute myocardial infarction. Int I Cardiol 2006; I I : 247-255.

22. Levick SP, Goldspink PH. Could interferon-gamma be a therapeutic target for treating heart failure? Heart Fail Rev 2014; 19: 227236.

23. Battistoni A, Rubattu S, Volpe M. Circulating biomarkers with preventive, diagnostic and prognostic implications in cardiovascular diseases. Int J Cardiol 2012; 157: 160-168.

24. Mata-Espinosa DA, Hernández-Pando R. Interferón gamma: aspectos básicos, importancia clínica y usos terapéuticos. Rev Invest Clin 2008; 60: 421-431.

25. Maiorana C, Duque J, Pellino M, Albiarez I, Lares M. Niveles plasmáticos de péptido natriurético cerebral (NTproBNP), Proteína C Reactiva Ultrasensible (PCRus) y su relación con diámetros de cavidades del ventrículo izquierdo en pacientes con insuficiencia cardiaca. Rev Latinoam Hiperte 2012; 7: 7- 12.

26. Bautista LE, Vera LM, Arenas IA, Gamarra G. Independent association between inflammatory markers (C-reactive protein, interleukin-6, and TNF-alpha) and essential hypertension. J Hum Hypertens 2005; 19: 149- 154. 